\title{
A Heterogeneous Traffic Virtual-Reality Simulator to Study Irritation/Anger and Driving Behavior under Adverse Conditions
}

\author{
Mayank Agrawal \\ IIIT Hyderabad \\ mayank.agrawal@research.iiit.ac.in
}

\author{
Kavita Vemuri \\ IIIT Hyderabad \\ kvemuri@iiit.ac.in
}

\begin{abstract}
To analyse driver's behaviour in heterogeneous chaotic traffic, simulators are important as experimentation in real traffic is dangerous. Simulators using Virtual Reality HMD provide a 360degree view enabling almost realistic experience. In our research, we focus on studying the driver's response when forced to follow a slow-moving vehicle with or without lane regulation. We collected self-reports of emotions - anger/frustration. Set as a first-person view through the windshield, a player controls the virtual car in simulated (game engine Unity3D) traffic with a steering wheel fitted with hand operated acceleration and brake hardware controls. Road scenarios similar to a typical Indian condition are replicated and each individual vehicle type is implemented as Artificial Intelligent (Al) bot. These bots interact with the player forcing her/him to react to the emergent situations. The player's goal is to overtake a very slow-moving vehicle ahead blocking the traffic and the scenarios presented were: a) no-lanes, b) lane change possible only at certain stretches of the road and c) lanes demarcated by solid barricades. Traffic sounds were added for natural effect. Participants with and without real-life driving experience were recruited. The time to reach the finish line was the least in the no-lane condition and the driver was able to manoeuvre through the gaps between vehicles. Designated lane-change stretches required quick responses and speed-prediction skills, in the absence of which collisions were observed and also time to finish was longer. Though lane marking and discipline is promoted for safety, in heterogeneous traffic with vehicles of varying engine capacity, strict lane adherence could lead to massive traffic jams and driver frustration/road rage.
\end{abstract}

Virtual Reality, heterogeneous traffic, lane, slow moving vehicles, stereophonic sound, simulation.

\section{INTRODUCTION}

A driving simulator is a virtual reality, which includes realistic vehicle dynamics: audio, immersive video and kinetics systems (like 6-DoF platform) emulating vehicle motion, surroundings scenes and traffic noises. A setup such as this can be used efficiently for testing vehicle safety in crash testing, effects on driving due to changes in the external traffic system and most importantly in human factor studies. Virtual simulators offer many advantages over real world experiments, some of them being, replication, experimental control, postdiagnosis and safe-testing of variants in traffic scenarios. Hence, driving simulators are important tools which allow the researcher to manipulate the situations [1][2], a popular commercial product is the VISSIM(software package developed by PTV Planning Transport Verkehr AG in Karlsruhe, Germany) to analyse traffic flow.

Modelling and testing driving behaviour in heterogeneous and low-discipline adherence traffic requires flexibility to study culture restricted behavioural study, for example, variable pedestrian behaviour, random changes in road conditions and irrational driving behaviour. Research on modelling traffic flow at a micro-macro level has been exhaustive for homogeneous vehicle type for which disciplined lane traffic and robust models have been proposed. But in the case of no-lane (where no lanes are marked) or weak-lane discipline traffic conditions, with multiple transient leaders and hence lateral interactions exist, new models are required. A few studies [3-6] have looked at weak lane discipline traffic and lateral interactions. A comprehensive review [5] of the models for carfollowing and lane-changing note the advantages and shortcomings for non-regulated traffic conditions. Studies on acceleration decision making [7], complexity from smaller vehicles [8], fitting European models to Asian traffic conditions [9], lane discipline [10][11] and behaviour in heterogeneous traffic [12] have been analysed. A review [13] of the various microscopic driving models and the applicability to Indian traffic types states that these models do not consider all the complexities of heterogeneous traffic and the dynamic strategies drivers apply. 
The roads in India carry buses, trucks, 3-wheeler public and goods vehicles, 2-wheeler motorbikes or scooters, cars of different engine capacity, nonmotorized push-carts/bicycle, pedestrians and animals. To follow lane discipline is almost impossible in India as there is inconsistency in each vehicle's capacity and power. There are situations where fast moving vehicles are forced to follow a slow-moving vehicle. If there is dense and fast traffic on both sides of the fast-moving vehicle, then a higher engine capacity vehicle has to follow a slow vehicle with engine capacity below allowed speed-limits. This scenario is considered in this study and behaviour (frustration/irritation) and congestion effects are analysed. Hence, the focus of this study is to understand the participant's decisions on discretionary lane change to overtake an immediate leading vehicle, which is a slowmoving car/autorickshaw by setting three scenarios: a) no lanes, b) designated lane changing stretches and c) barricaded lanes. This has been explained in the following section followed by results and discussion.

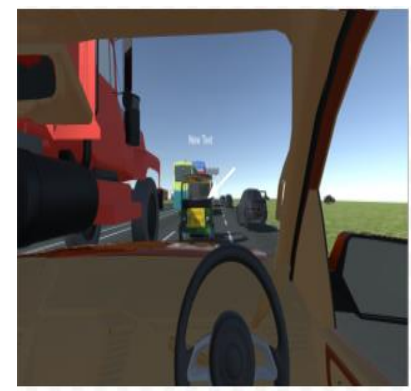

Figure 1(a)

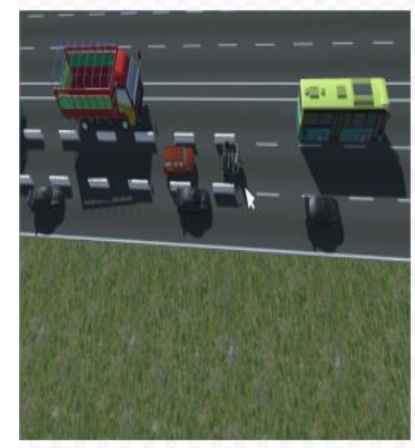

Figure $1(c)$

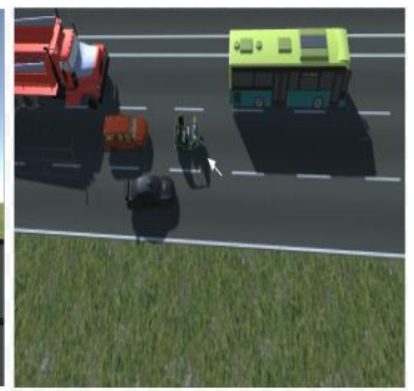

Figure 1(b)

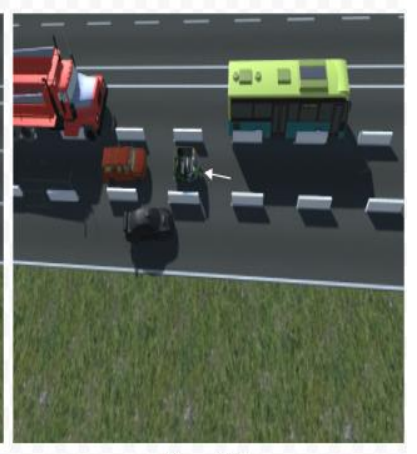

Figure 1(d)
Figure 1: (a) first-person-view of the road where the participant is the driver, (b) without lane barriers,(c)road with half lane barrier, (d) road with full lane barrier. Slow moving vehicle is marked with white arrow.

\section{SCENARIOS}

Three scenarios were created for the testing. Figure 1a shows the driver's first-person view through the wind-shield. The participant has to cover a half kilometre distance to reach the end point and then the timer will stop. In scenario 1 (Figure 1b), a two-stroke engine slow-moving three-wheeler autorickshaw is in front of the participant's car. Both are in the same middle lane. There are heavy vehicles which are moving on the left lane and cars which are moving on the right side of this lane. The participant can overtake from either side and also from the gap between twovehicles. In scenario 2 (Figure 1c), the participants can overtake or change lanes only at designated stretches. In the simulator, physical barricades were placed to indicate no-lane-change stretch. The driver can estimate the speed of the vehicles on the two side lanes and shift to the corresponding lane in the stretch of the road where it is allowed. In the last test setup (Figure 1d), The car is forced to follow the autorickshaw for the whole journey due to the physical barricades (Figure 1d). As all the participants are following the same slowly-moving autorickshaw for the entire road, their timings are almost same, but this condition was introduced to specifically gauge the frustration or anger levels.

\section{THE HETEROGENEOUS TRAFFIC SIMULATOR DESIGNED AND DEVELOPED}

A simulation was designed in-house and developed using Unity 3D game studio for ease of customization to fit the constraints and conditions of heterogeneous traffic and the application was formatted for virtual reality head mounted display, the Oculus Rift. The virtual car was controlled by a steering wheel hardware accessory fitted with hand-operated accelerator and brake. The scenarios were presented to the player in a 360degree view of a 3 lane road with trucks, buses, cars and autorickshaw programmed using simple rule-based artificial intelligence. These Al bots had characteristics of the type of vehicle represented, and behaviour models usually found on Indian roads. The heavier vehicles were positioned on the left lane while the participant's car is in the middle lane and the right lane was populated with car-bots. The two wheelers are mixed in between the lanes as is usually the condition on the Indian roads. Rigid-body collision detection was introduced to detect collision between the vehicles. Naturalistic ambient stereophonic traffic sounds and intermediate honking were also implemented. As we are interested in looking at driver's behaviour, when the lead in the lane is a very slow moving vehicle, a three-wheeler autorickshaw was considered for this role. The setup was designed to give the participant a first-person driver's perspective (Figure 1a). Three scenarios as listed above were presented and driver behaviour - time 
to reach the finish lane, decisions to change lanes or overtake - is recorded. The data recorded includes the Al bots positional coordinates with reference to the participant's vehicle and driving decision changes are event logged. Before the main experiment, all participants were allowed to get accustomed to the VR environment and control gear.

\section{RESULTS}

A total of 30 participants were recruited for the study. The participant age group ranged between 19 years to 25 years as shown in Table 1 . Out of these people, $56.7 \%$ people knew how to drive. $36.7 \%$ were female participants.

Table 1: Number of people distributed over age.

\begin{tabular}{|l|l|}
\hline Age & No of people (\% female) \\
\hline 19 & $2(50 \%)$ \\
\hline 20 & $4(50 \%)$ \\
\hline 22 & $8(75 \%)$ \\
\hline 23 & $12(66 \%)$ \\
\hline 24 & $4(25 \%)$ \\
\hline
\end{tabular}

The task was explained to the participants and trials were conducted to get the visuo-motor coordination in using the steering wheel, accelerator and brake function. Immersive visualization and interaction parameters to evaluate the virtual traffic simulation prototype were rated on a 1-5 scale, with the greater number indicative of the maximum sensation of reality. The distribution (Figure 2) indicates that 13 of the 30 participants found the prototype immersive, while an equal number were neutral $(3 / 5)$ and only 4 gave lower score $(2 / 5)$.

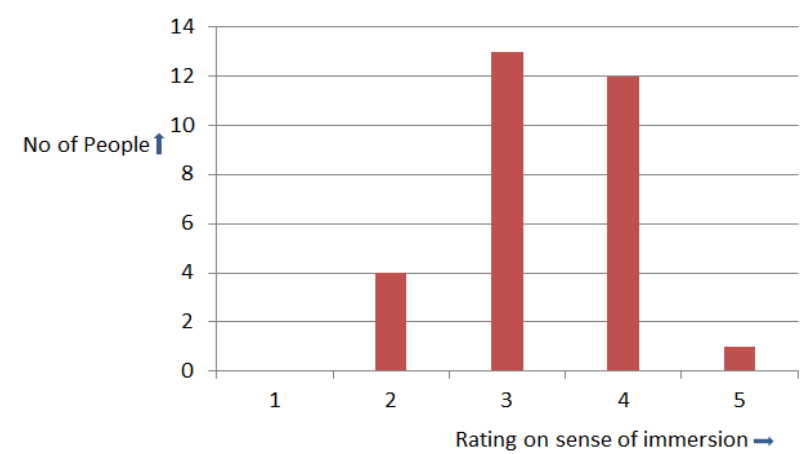

Figure 2: The rating on sense of immersion on a likely scale of $1-5(x$-axis) with 5 being maximum. The $y$ axis denotes the number of participants

The ratings are encouraging to the application of VR/tactile interactions for traffic behaviour testing, but also point to changes required in the application to increase the immersion. One reason for the lower rating could be motion sickness as VR was first time experience for many. We need to conduct re-tests to check whether this is a confounding factor.

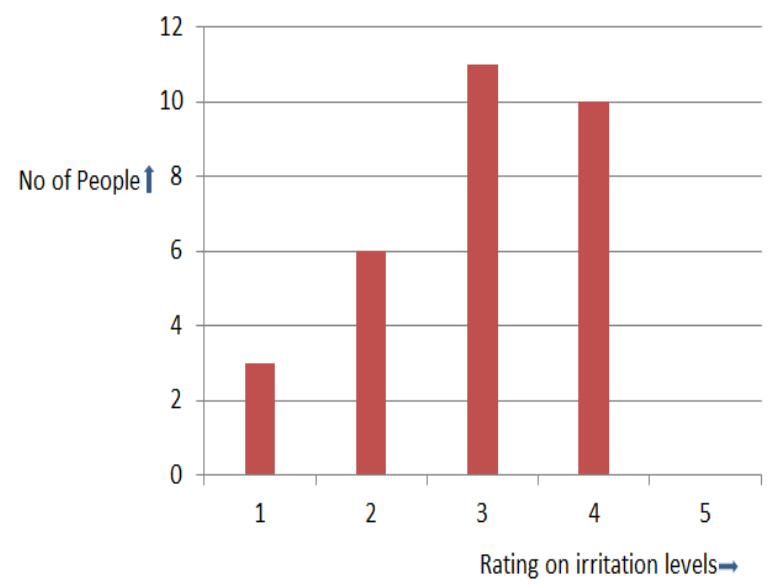

Figure 3: The rating on irritation levels on a scale of 1-5 (x-axis) with 5 being the maximum. The $y$-axis denotes the number of participants.

The ambient traffic noise along with frequent loud honking and the forced following of a slow-moving vehicle obstructing their path lead to irritation or anger. This is an index of driver's aggressive behaviour leading to erratic lane change decision making. The histogram shown in Figure 3 presents the distribution and as can be inferred the irritation level was reasonably high in 21 (3/5 rate and 4/5 rate) participants. This rating was collected only once at the end of testing for all scenarios and is combined effect of noise levels and the traffic conditions encountered. The participants reported that the irritation level was the highest when lane change was not possible due to the physical barricades, as they were restrained to follow the slow-vehicle until the end.

The average time for each scenario was calculated for all the participants (Table 2). A 2-sample T-test was applied to look at statistical significance for the timings from the first two scenarios with 95\% confidence level. The $p$ value comes out to be less than 0.0001 . The standard error of difference is 3.875. The statistical significance was estimated only for scenarios 1 and 2, because for the third scenario the time to reach the finish line was the speed programmed for the slow-moving vehicle by default.

Table 2: The average time taken by the 30 participants to navigate through the simulation for each scenario presented in the experiment.

\begin{tabular}{|c|c|}
\hline Scenario & $\begin{array}{c}\text { Avg time to reach } \\
\text { destination (sec) }\end{array}$ \\
\hline
\end{tabular}




\begin{tabular}{|l|l|}
\hline $1-$ no lane & 38.7 \\
\hline $\begin{array}{l}2 \text { - specific stretch for } \\
\text { lane change }\end{array}$ & 334 \\
\hline $\begin{array}{l}3 \text { - physical barricades } \\
\text { for lanes }\end{array}$ & 645 \\
\hline
\end{tabular}

\section{DISCUSSION}

From the results we can infer that the virtual reality setup developed for this experiment was efficient for behavior testing and also for understanding traffic flow, especially in heterogeneous traffic. The reflex shock response to an accident in the VR environment was reported by some participants. The immersion rating is reasonable and we interpret the participants who gave lower rating to be either due to limitations in the computer graphics and/or motion sickness [14][15]. Another important limiting factor could be the interface, a real-car body with actual steering wheel and accelerator/brake system would have increased the immersion. The time to reach the finish lane in each of the scenarios was as expected within acceptable error bar. That is, due to the response time constraints imposed by the VR glasses in field-of-view and the haptic feedback from the steering wheel. However, a study [16] that compared the reaction time in the virtual simulator and in real driving experiment found the results were near similar for both conditions with reactions times in the virtual simulator being just slightly better than in the real-driving experiment.

In the simulator, the high decibel traffic noise input, the frustrations from following a slow-moving vehicle and inability to change lanes induced high level of irritation/anger in $73 \%$ of the participants (rating $>=3$ ). The overall lower rating on this index could the consciousness of being in a virtual world or subjective variation in tolerance to noise levels or threshold for anger/irritation. At the same time it should also be acknowledged that some behaviors in the virtual world might be more aggressive than in real-life as the fear of physical harm is not accounted for.

We interpret the results to indicate the simulation was realistic to the level that participants felt they had control over the car and attempted to navigate the traffic to reach the finish line. The results on time taken to reach the finish lane for each lane condition show that simulations like these can be used to test the effects due to introduction or removal of new rules, thus, establishing VR, as a visualization medium for road/traffic planning and gauging citizen responses. The experiment to rate irritation/anger is important as it shows driver accumulated aggression leading to road rage in tiring situations. The setup used is also a novel method to measure irritation which could help in interventions or preventive measures to reduce anger levels in drivers.

\section{LIMITATIONS}

One major limitation is the number of participants who have longer driving experience. As all shortcomings of simulators [18], ours too has some limitations in terms of realistic rendering. We acknowledge that simulators can also produce unrealistic driving behavior and therefore can lead to false research results.

\section{REFERENCES}

1. Meuleners, L., \& Fraser, M. (2015). A validation study of driving errors using a driving simulator. Transportation research part F: traffic psychology and behaviour, 29, 14-21.

2. Shechtman, O., Classen, S., Awadzi, K., \& Mann, W. (2009). Comparison of driving errors between on-the-road and simulated driving assessment: a validation study. Traffic injury prevention, 10(4), 379-385.

3. Gunay, B. (2008). A methodology on the automatic recognition of poor lane keeping. Journal of advanced transportation, 42(2), 129-149.

4. Ravishankar, K. V. R., \& Mathew, T. V. (2011). Vehicle-type dependent carfollowing model for heterogeneous traffic conditions. Journal of transportation engineering, 137(11), 775-781.

5. Mahapatra, G., \& Maurya, A. K. (2013). Study of vehicles lateral movement in nonlane discipline traffic stream on a straight road. Procedia-Social and Behavioral Sciences, 104, 352-359.

6. Metkari, M., Budhkar, A., \& Maurya, A. K. (2013). Development of simulation model for heterogeneous traffic with no lane discipline. Procedia-Social and Behavioral Sciences, 104, 360-369.

7. Choudhury, C. F., \& Islam, M. M. (2016). Modelling acceleration decisions in traffic streams with weak lane discipline: A latent leader approach. Transportation research part C: emerging technologies, 67, 214-226.

8. Nair, R., Mahmassani, H. S., \& MillerHooks, E. (2011). A porous flow approach to modeling heterogeneous traffic in disordered systems. Transportation Research Part B: Methodological, 45(9), 1331-1345. 
9. Lenorzer, A., Casas, J., Dinesh, R., Zubair, M., Sharma, N., Dixit, V., \& Brackstone, M. (2015, September). Modelling and Simulation of Mixed Traffic. In Australasian Transport Research Forum (ATRF), 37th, 2015, Sydney, New South Wales, Australia.

10. Maurya, A. K. (2011). Comprehensive approach for modeling of traffic streams with no lane discipline. In 2nd International Conference on Models and Technologies for Intelligent Transportation Systems.

11. Gandla, V. V., \& Santhakumar, M. NONLANE-BASED MICROSCOPIC MODELLING UNDER HETEROGENEOUS TRAFFIC CONDITIONS.

12. Munigety, C. R., \& Mathew, T. V. (2016). Towards Behavioral Modeling of Drivers in Mixed Traffic Conditions. Transportation in Developing Economies, 2(1), 6.

13. Asaithambi, G., Kanagaraj, V., \& Toledo, T. (2016). Driving Behaviors: Models and Challenges for Non-Lane Based Mixed Traffic. Transportation in Developing Economies, 2(2), 19.

14. Brooks, J. O., Goodenough, R. R., Crisler, M. C., Klein, N. D., Alley, R. L., Koon, B. L., ... \& Wills, R. F. (2010). Simulator sickness during driving simulation studies. Accident Analysis \& Prevention, 42(3), 788-796.

15. Kolasinski, E. M. (1995). Simulator Sickness in Virtual Environments (No. ARI-TR-1027). ARMY RESEARCH INST FOR THE BEHAVIORAL AND SOCIAL SCIENCES ALEXANDRIA VA.

16. Riener, A. (2010, February). Simulating onthe-road behavior using a driving simulator. In Advances in Computer-Human Interactions, 2010. ACHI'10. Third International Conference on (pp. 25-31). IEEE. 